\title{
Periodontal Status Indices and Risk of Psoriasis in a Greek Adult Population: A Case - Control Study
}

\author{
Nikolaos Andreas Chrysanthakopoulos ${ }^{*}{ }^{1}$, Eleftheria Vryzaki ${ }^{2}$ \\ ${ }^{1}$ Dental Surgeon (DDSc), Oncologist (PhD), Specialized in Clinical Oncology, Cytology and Histopathology, Dept. of \\ Pathological Anatomy, Medical School, University of Athens, Athens, Greece \\ ${ }^{2}$ Dermatologist (MD), PhD, University Hospital of Patra, Greece, Periodontal disease indices and Psoriasis \\ Association
}

Received: February 26, 2021; Accepted: March 15, 2021; Published: April 19, 2021

*Corresponding author: Nikolaos Andreas Chrysanthakopoulos 35, Dental Surgeon (DDSc), Oncologist (MSc), Specialized in Clinical Oncology, Cytology and Histopathology, Dept. of Pathological Anatomy, Medical School, University of Athens, Athens, Greece, Tel./Fax. No: 0030-2610-225288; E-mail: nikolaos_c@hotmail.com,nchrysant@med.uoa.gr

\begin{abstract}
Few researches have investigated the potential role of periodontal disease as a risk factor for developing psoriasis. However, the possible link remains still unidentified.

Aim: The aim of the current study was to investigate the possible relationship between periodontal status indices and the risk for psoriasis development in a Greek adult population.

Subjects and Methods: The study sample comprised 337 psoriasis patients-cases and 337 healthy individuals-controls between 40 to 69 years of age who referred by three dermatology and one dental private practice. Cases and controls completed a health medical and dental questionnaire and underwent an examination of their periodontal status that included the following parameters: Probing Pocket Depth (PPD), Clinical Attachment Loss (CAL), frequency of a regular/irregular annual dental follow-up, and number of missing teeth. Odds ratios (OR's) and 95\% Confidence Intervals (95\% Cl's) were assessed using logistic regression model adjusted for possible confounders.

Results: The multivariate regression analysis model showed that a higher BMI ( $\mathrm{p}=0.009,0 \mathrm{R}=3.154,95 \% \mathrm{CI}=1.532-4.028)$, alcohol overconsumption $(\mathrm{p}=0.012$, OR=2.024, 95\% CI=1.445-3.435), moderate /severe CAL ( $\mathrm{p}=0.047,95 \% \mathrm{CI}=2.112,1.156-3.249)$, and a number of missing teeth more than four $(\mathrm{p}=0.027, \mathrm{OR}=2.817,95 \% \mathrm{CI}=1.267-3.872$ and $\mathrm{p}=0.016, \mathrm{OR}=3.510,95 \% \mathrm{CI}=1.350-4.145)$ were statistically significantly
\end{abstract} associated with risk for psoriasis development.

Conclusion: Individuals with a higher BMI, excessive alcohol consumption, moderate/severe attachment loss and a number of missing teeth more than four were at significantly higher risk for developing psoriasis.

Keywords: Periodontal disease; Psoriasis; Number of missing teeth; Risk factor.

\section{Introduction}

Psoriasis is a chronic inflammatory skin disease affecting $1-3 \%$ of the population [1], whereas its prevalence ranges from 2 to $4 \%$ in the general population [2]. The course of the disease is chronic, characterized by exacerbations and relapses, dermis and epidermis chronic inflammation that is associated with sharply-demarcated papules and plaques, markedly thickened epidermis and atypical keratinocyte differentiation [1]. The etiology of the disease still remains unknown; however previous researches have proposed several risk factors including smoking, high body mass index (BMI) [3-5], sedentary life-style and low physical activity [6], and high alcohol consumption [7-10]. Other reports support that genetic predisposition, physical traumas, various medications, stress [11] and infectious factors [12] are able to trigger and exacerbate the disease [13], whereas it seems that gender predisposition is not involved in its etiology[2]. Periodontal Disease (PD), gingivitis and mainly periodontitis, is a chronic inflammatory disease of the periodontal tissues which without treatment can lead to deep pocket formation, loss of the bone, teeth mobility, and teeth losses [14]. PD is a common chronic disease that affects approximately one-third of adults over 30 years of age and up to half of adults over 50 years of age $[15,16]$ and it has also been estimated that affects 30\%-35\% of dentate US adults $[17,18]$. Known risk factors of PD include poor oral hygiene, infrequent brushing habits, immune status, smoking, stress, and genetic predisposition[19-21], increasing age[22], male gender [23,24], educational level, socio-economic status, [25-27], social and psychological factors [28], and diabetes mellitus $[24,25]$. Several microorganisms and products on the dental plaque are responsible for the initiation and progression of $\mathrm{PD}$, as it represents an exaggerated inflammatory response to polymicrobial colonization in the dental plaque $[29,30]$. PD is associated with a systemic inflammatory response, increasing levels of inflammatory cytokines, chemokines and 
other inflammatory mediators such as C-reactive protein (Crp), Interleukin-1(IL-1) and IL-6 [29], that are responsible for periodontal tissues damage [31]. PD has also been associated with several systemic diseases and disorders, such as diabetes mellitus, cardiovascular disease, respiratory disease, inflammatory bowel disease [32] cancer [33] and systemic infections [34,35]. An association between PD and immune-mediated inflammatory diseases has been increasingly identified, as it has been supposed that oral cavity bacterial colonization could induce an overactive immune response in a susceptible host, leading to a lasting inflammatory process associated with autoimmune disorders [36]. Previous reports have been most extensively investigated the mentioned association in Rheumatoid Arthritis (RA), and found that the odds of having RA among those with periodontitis was 2 to 8 times higher than those without periodontitis [3741]. Both diseases, psoriasis and PD implicate an exaggerated immune response in epithelial surfaces and an abnormality in the regulation of the host inflammatory response $[11,42,43]$. A previous blinded case-control study revealed a significantly lower radiographic bone level $(\mathrm{p}<0.001)$ and a significantly higher number of missing teeth $(p<0.001)$ in the psoriasis cases compared to their age-and gender-matched controls [11]. Moreover, previous case-reports and original articles showed that psoriasis exacerbation accompanying aggravated periodontitis [44],whereas after periodontitis treatment with topical antibiotics, tooth extraction, and shaping of alveolar bone regression of palmoplantarpustulosis was observed $[45,46]$. There are no previous prospective or retrospective studies estimating the possible association between PD and psoriasis. In this study, we hypothesized that those with PD, as measured by clinical assessing of PD indices, were at greater risk for subsequent psoriasis, in a sample of Greek adults. Patients with periodontitis may also have a higher risk of psoriasis, however, the data on this association are limited. The aim of this retrospective case-control study was to further analyze the possible association between periodontitis and psoriasis

\section{Subjects and Methods}

\section{Study design}

A retrospective case-control, epidemiological study was performed between 2018 and 2020. The study size, 337 cases and 337 controls, was assessed based on the Psoriasis prevalence determined by Hyman et al. [47], with 95\% confidence interval and $1.0 \%$ range of error, whereas the age group was based on the World Health Organization (WHO) recommendations [48,49] for assessing disease prevalence.

Psoriasis patients-cases and healthy individuals-controls, referred by three dermatology and one dental private practice completed a health medical and dental questionnaire and their periodontal status was examined by a well-trained and calibrated dental surgeon. Individuals who had less than 20 natural teeth, those who were treated for PD, conservatively or surgically, within the previous six months and those who had received a prescription of systemic antibiotics or anti-inflammatory or other systemic drugs, such as glucocorticoids the previous six weeks were not enrolled in the study protocol as those conditions could influence [50] the oral tissues condition, and could lead to biased secondary associations. Cases group diagnosis was based on their medical files. Controls group was consisted of individuals from the cases' friendly and collegial environment in an effort to avoid potential confounding factors such as age, gender, smoking and SES.

\section{Data collection}

Cases and controls filled in a modified Minnesota Dental School Medical Questionnaire [51] that included epidemiological parameters such as age, gender, smoking status, alcohol intake, socioeconomic and educational status, Body Mass Index (BMI), current diseases and disorders, past medical/dental and family history.

Participants' age was classified as 40-49, 50-59, 60-69; socioeconomic status as $\leq 1,000$ and $>1,000 € /$ month; educational status as elementary level and graduated from University/ College; smoking status as never smokers and former/current smokers; and frequency of a regular dental follow-up as $\leq 2$ examinations/year and $>2$ times/year.

Probing Pocket Depth (PPD) index was classified as 0-3.00 mm (absence of disease/mild disease) and $\geq 4.0 \mathrm{~mm}$ (moderate and severe disease) for mean PPD [52], attachment loss (CAL) severity was classified as mild, 1-2.0 mm of attachment loss and moderate/severe, $\geq 3.0 \mathrm{~mm}$ of attachment loss [53], and tooth loss as none, 1-4, 5-10, >10 missing teeth [54].

\section{Periodontal status examination}

Periodontal status indices were estimated at six sites in all teeth (mesio-buccal,disto-buccal, mid-buccal, mesio-lingual distolingual, and mid-lingual), excluding third molars, and remaining roots using a manual periodontal probe (UNC-15; Hu Friedy Mfg. Co. Inc., Chicago, IL USA).

A standard number of 28 teeth was used as reference and assessed the existed number of teeth, and then minus the number of existed teeth from 28 teeth, and the outcome this was defined as number of lost teeth or missing teeth. Missing teeth caused by other conditions such as accidents or orthodontic treatment were not included in the statistical analysis. For assessing the intraexaminer variance a randomly selected sample of 135 (20\%) individuals

was reexamined clinically by the same dentist after 3 weeks. No differences were observed after the second clinical examination (Cohen's Kappa=0.94). 


\section{Statistical analysis}

For each participant, patient and healthy the mean value of PPD and CAL at the mentioned sites was measured, the measurements concerned the immediate full millimeter, and classified as dichotomous variables. Females, lower socio-economic (income/monthly $\leq 1,000 €$ ) [55] and educational status individuals, never smokers, those with an irregular dental follow-up, individuals with BMI $<30$ [56] and those with alcohol intake $<32 \mathrm{~g}$ or $24 \mathrm{~g}$ of alcohol for males and females/daily respectively, and/or weekly $168 \mathrm{~g}$ of alcohol for males and 112g for females [57] were classified as 0. Individuals with absence of disease/mild disease (PPD:0-3.00 mm) and those with mild attachment loss (CAL 1-2.0 mm) were classified as 0 . The missing teeth were classified as $0,1,2$, and 3 for number of missing teeth none- $0,1-4,5-10$, and $>10$, respectively.

Chi-square test (univariate analysis) was carried out for assessing the associations between Psoriasis and demographic/clinicalindices or potential risk factors. The enter method of multivariate regression analysis model was carried out to estimate the associations between the dependent variable, Psoriasis, and independent ones. The stepwise method was performed in order to assess gradually the variables that had significant associations with the dependent one.

Unadjusted (UOR's) and Adjusted Odds Ratios (AOR's) and 95\% CI (Confidence Interval) were also assessed. All statistical analyses were carried out using SPSS statistical package (SPSS PC19.0, SPSS, Inc., Chicago, IL,USA). A p-value of < 0.05 was considered statistically significant.

\section{Ethical consideration}

The current cross-sectional study was carried out in full accordance with the World Medical Association Declaration of Helsinki and was not an experimental one. In Greece only experimental studies must be reviewed and approved by authorized committees (Dental

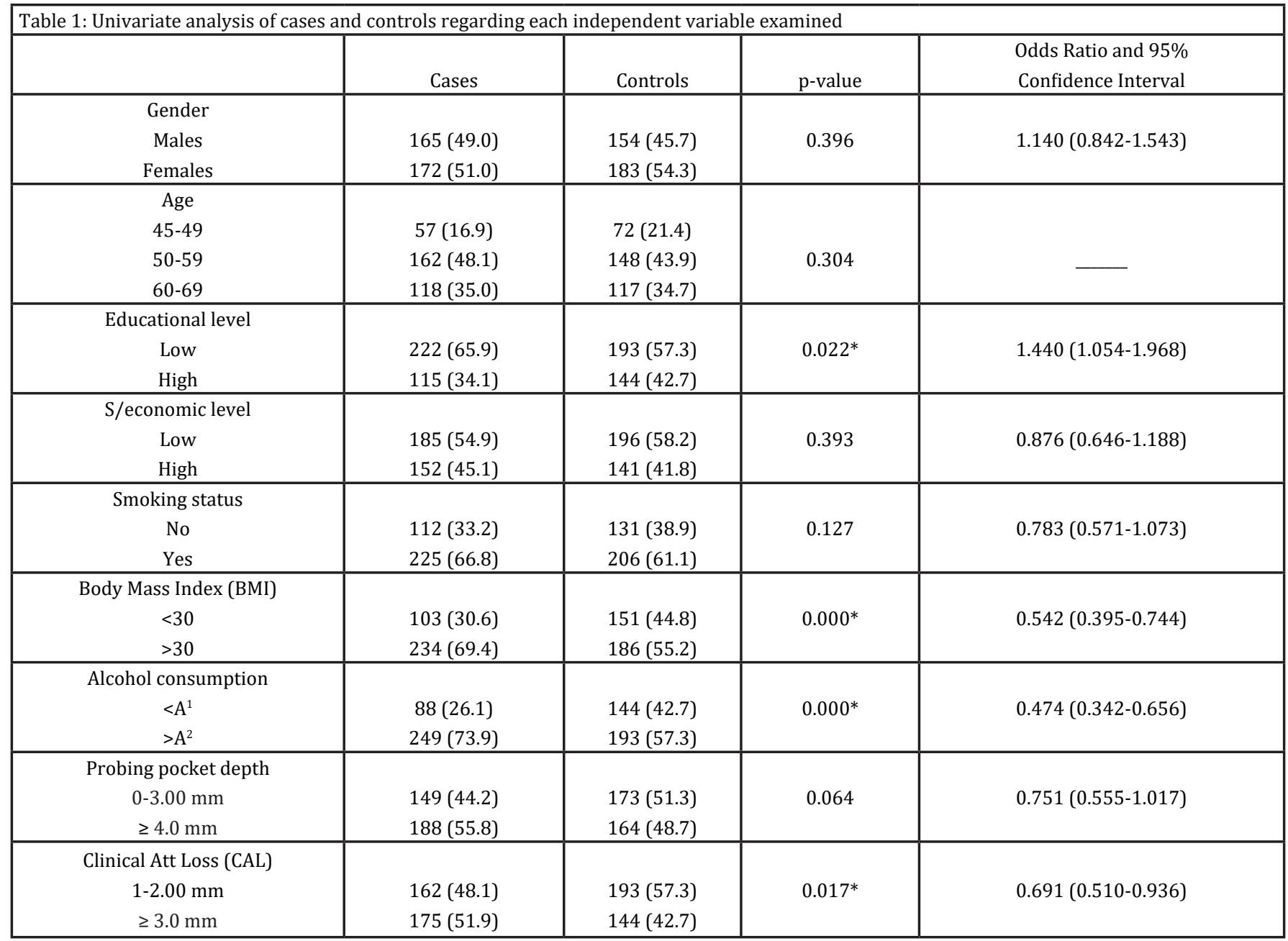




\begin{tabular}{|c|c|c|c|c|}
\hline $\begin{array}{l}\text { Dental Follow-up } \\
\leq 2 \text { times/annually } \\
>2 \text { times/annually }\end{array}$ & $\begin{array}{l}142(42.1) \\
195(57.9)\end{array}$ & $\begin{array}{l}153(45.4) \\
184(54.6)\end{array}$ & 0.393 & $0.876(0.646-1.188)$ \\
\hline $\begin{array}{c}\text { Tooth loss } \\
\text { None } \\
1-4 \\
5-10 \\
>10\end{array}$ & $\begin{array}{c}26(7.7) \\
61(18.1) \\
103(30.6) \\
147(43.6)\end{array}$ & $\begin{array}{c}33(9.8) \\
77(22.8) \\
125(37.1) \\
102(30 .)\end{array}$ & $0.050^{*}$ & - _ - \\
\hline
\end{tabular}

Schools, Greek Dental Associations, Ministry of Health, etc). Individuals who accepted the invitation to be enrolled in the study protocol signed an informed consent protocol.

\begin{tabular}{|c|c|c|c|c|c|c|c|c|c|}
\hline & & \multirow[b]{2}{*}{ B } & \multirow[b]{2}{*}{ S.E. } & \multirow[b]{2}{*}{ Wald } & \multirow[b]{2}{*}{$\mathrm{df}$} & \multirow[b]{2}{*}{ Sig. } & \multirow{2}{*}{$\begin{array}{l}\operatorname{Exp}(B) \\
\text { Lower }\end{array}$} & \multicolumn{2}{|c|}{ 95\% C.I.for EXP(B) } \\
\hline & & & & & & & & Upper & \\
\hline \multirow[t]{15}{*}{ Step $1^{\mathrm{a}}$} & gender &,- 115 & 167 & ,473 & 1 & ,492 & ,892 & 643 & 1,236 \\
\hline & age & ,125 & 117 & ,082 & 1 & 467 & 1,005 & ,799 & 1,263 \\
\hline & socioecon.stat & ,288 & 173 & 2,759 & 1 & ,097 & 1,334 & ,949 & 1,874 \\
\hline & educ.level & 102 & 166 & ,024 & 1 & ,878 & 975 & ,703 & 1,351 \\
\hline & smok.stat & ,179 & ,172 & 1,080 & 1 & ,299 & 1,196 & ,853 & 1,677 \\
\hline & bod.mas.ind & ,382 & 197 & 4,557 & 1 &, $014^{*}$ & 2,431 & 1,577 & 3,756 \\
\hline & alcohol.intake &, 340 & ,185 & 4,112 & 1 &, $021^{*}$ & 2,075 & 1,471 & 2,927 \\
\hline & dent.fol.up & 094 & 172 & ,297 & 1 & ,586 & 911 & ,650 & 1,275 \\
\hline & prob.pock.dep & 112 & 189 & ,094 & 1 &, 550 & 1,012 & 698 & 1,466 \\
\hline & clin.att.loss & ,293 & ,163 & 3,057 & 1 &, $048^{*}$ & 1,670 & 1,144 & 2,439 \\
\hline & tooth.loss & & & 2,793 & 3 & ,085 & & & \\
\hline & tooth.loss(1) & 277 & ,145 & 2,722 & 1 &, 064 & 1,124 & 0,722 & 1,610 \\
\hline & tooth.loss(2) &, 285 & , 185 & 3,187 & 1 &, $039 *$ & 2,413 & 1,261 & 3,254 \\
\hline & tooth.loss(3) & ,301 & 189 & 3,799 & 1 &, $012^{*}$ & 3,522 & 1,354 & 3,769 \\
\hline & Constant & 1,915 & 331 & 7,622 & 1 & ,031 & ,401 & & \\
\hline \multirow[t]{8}{*}{ Step $8^{a}$} & bod.mas.ind & ,467 & ,214 & 4,505 & 1 &, $009 *$ & 3,154 & 1,532 & 4,028 \\
\hline & alcohol.intake & ,495 & 197 & 4,814 & 1 &, $012^{*}$ & 2,024 & 1,445 & 3,435 \\
\hline & clin.att.loss & ,478 & ,170 & 3,916 & 1 &, $047^{*}$ & 2,112 & 1,156 & 3,249 \\
\hline & tooth.loss & & & 3,347 & 3 & ,066 & & & \\
\hline & tooth.loss(1) & 634 & ,317 & 3,493 & 1 & ,052 & 1,531 & 0,851 & 1,688 \\
\hline & tooth.loss(2) &, 874 & ,328 & 4,156 & 1 &, $027^{*}$ & 2,817 & 1,267 & 3,872 \\
\hline & tooth.loss(3) & ,673 & ,363 & 4,225 & 1 &, $016^{*}$ & 3,510 & 1,350 & 4,145 \\
\hline & Constant & 1,721 & ,220 & 8,728 & 1 & ,011 & ,486 & & \\
\hline
\end{tabular}

*p-value: statistically significant 


\section{Results}

The study sample consisted of 337 cases, 155 males and 182 females and 337 controls, 164 males and 173 females, with a mean age 58.2 years $( \pm 2.4)$. The epidemiological parameters of psoriasis patients and healthy individuals after performing the univariate analysis are shown in Table 1. Lower educational level $(\mathrm{p}=0.022)$, increased BMI $(\mathrm{p}=0.000)$, increased alcohol consumption $(\mathrm{p}=0.000)$, moderate/severe CAL $(\mathrm{p}=0.047)$, and increased number of missing teeth were statistically significantly associated with risk for psoriasis development. Table 1 also presents Unadjusted Odds Ratio and 95\% Confidence Interval for each variable examined.

The multivariate regression analysis model (Enter and Wald methods) is presented in Table 2, in which also shown Adjusted Odds Ratio and 95\% are Confidence Interval for each variable examined. According to the Wald model the same indices were statistically significantly associated with risk for developing psoriasis.

\section{Discussion}

The present case-control study showed that individuals with a higher BMI and excessive alcohol consumption, moderate/ severe attachment loss ( $\geq 3.0 \mathrm{~mm}$ ) and a number of missing teeth more than four were at significantly higher risk for developing psoriasis. According to those findings it could be suggested that quitting alcohol intake and reducing BMI could be reduce the risk for the development of psoriasis. Nevertheless, more evidence is requires to confirm such observations.

No associations were recorded between common epidemiological parameters, such as gender, age, socio-economic status, educational level, smoking and psoriasis risk in the present research. Previous studies have also not confirmed such associations for their potential role in psoriasis aetiology, whereas it must be noticed that those epidemiological parameters act as confounders.

Alcohol overconsumption is associated with an increased risk of developing psoriasis $[7,58,59]$, finding that was in accordance with the current study. Alcohol consumption is able to affect innate and adaptive immunity and is an aggravating factor in inflammation response as increases the production of proinflammatory cytokines, such as tumor necrosis factor $-\alpha$ (TNF- $\alpha$ ) from peripheral blood monocytes and macrophages, and also increases lymphocyte proliferation and activation [60].

Experimental studies in mice have revealed that alcohol-induced liver injury can lead to damage of skin barrier unction, possibly mediated via plasma TNF- $\alpha$ [61], whereas it has also been shown that alcohol and can influence the number and function of epidermal $\mathrm{T}$ cells [62] and can reduce dendritic function in female mice [63]. Moreover, alcohol can influence migration of murine dermal dendritic cells to draining lymph nodes after sensitization [64].

Regarding the possible role of mast cells in psoriasis pathogenesis alcohol may affect the number of mast cells and their degranulation [65-67].

However, more research is required in order to determine whether alcohol can induce or worsen psoriasis [68].

BMI is another index that has been implicated in psoriasis aetiology. Previous reports have provided evidence that a higher BMI leads to a higher risk of psoriasis [3-5,69]. The outcomes of the current research confirmed the mentioned association.

It has been proposed that the increased production of inflammatory cytokines from adipose tissue and functional changes within adipose tissue are responsible for the potential link between obesity and skin inflammation [70]. Excess skin adipose tissue leads to pro-inflammatory cytokine and hormone secretion. TNF- $\alpha$ and IL- 6 are directly involved in psoriasis pathogenesis [71,72]. Moreover, leptin, and hormone that is regulates body weight can increase keratinocyte proliferation and pro-inflammatory protein secretion, that are biomarkers of psoriasis [73], whereas the adiponectin secretion which is a supposed anti-inflammatory factor [74], is reduced in obesity.

Obese individuals' skin shows signs of impaired barrier function, whereas dysregulation in lymphatic function may delay the clearance of the mentioned inflammatory mediators [70]. It is possible that other mechanisms are involved in the association examined; however they have not been investigated yet.

The current study showed that periodontal status indices, such as moderate/ severe attachment loss $(\geq 3.0 \mathrm{~mm})$ and a number of missing teeth more than four were associated with an increased risk for developing psoriasis.

A few numbers of studies have investigated the relationship between periodontal status and psoriasis in the literature. Preus et al. [11] in a case-control study found decreased alveolar bone levels in the psoriasis-cases group than controls. The same study showed that psoriasis patients had a higher number of missing teeth when compared to their age- and gender-matched controls ( $51 \%$ vs. $26 \%$, p < 0.001), without adjustment for confounders such as alcohol and smoking. In a more recent study was found a significant difference between the mean number of remaining teeth in cases and healthy individuals (24 vs. 26, respectively) [75]. Similarly, Akazawa et al. presented a female patient with severe periodontitis and psoriasis and highlighted that after her periodontal treatment the disease declined and did not relapse [45]. Ungprasert et al. in a metaanalysis[76] found a 1.55-fold increased risk of psoriasis among individuals with periodontitis compared with those without periodontitis. In the same report was found that the elevated risk was observed with both study designs, cohort and case-control, though the risk appeared to be higher among case-control studies. Similar results were confirmed by previous studies [77-80]. Those outcomes are in 
contrast with the findings of a research among nurses [81] in which was not recorded that individuals with fewer teeth (0-24 vs. 25-32) tooth loss history were at greater risk of psoriasis. The mechanisms by which PD may lead to greater risk of psoriasis remains unclear. As mentioned, the bacteria in the periodontal tissues are responsible for a chronic infection and increase the levels of pro-inflammatory cytokines IL-6, TNF- $\alpha$ and IL-1 $\beta$ in the periodontal tissues and serum [82-85]. PD involves a bacterial infection with gram negative bacteria mainly, that invade superficial and subginigival tissues [86]. Those periopathogens and their products may stimulate the psoriasis pathway directly or indirectly through a systemic inflammatory reaction that involves mediators such as the pro-inflammatory cytokine IL17 , which has been found to be increased in both psoriatic skin [87] and the gingiva of chronic periodontitis patients [88]. Preus et al. [11], suggested another possible mechanism implicating the innate immune system, which has been found to be involved in the pathogenesis of both psoriasis and PD and may influence inflammatory cells such as dendritic cells and Toll-like receptor (TLR) expression, predisposing patients to both psoriasis and PD $[89,90]$. Moreover it has been demonstrated an upregulation of TLR-2 in psoriatic skin [87] and in the periodontal tissue of patients with periodontitis [91], as well another study in the Yaa mouse model that showed an increase in TLR genes can induce autoimmunity [92]. The association between periodontitis and an increased risk of immune-mediated inflammatory diseases has been well investigated in Rheumatoid Arthritis (RA). Porphyromonasgingivalis, a common gram negative anaerobic periopathogen, is the crucial link between RA and periodontitis because produces peptidyl arginine deiminase, the major enzyme that promotes post-translational citrullination of peptides. Excessive amount of those peptides could then induce production of Anti-Citrullinated Peptide Antibody (ACPA), which is an essential autoantibody in the pathogenesis of synovitis in RA $[40,93]$. Despite this observation, it is improbable that excessive citrullination of peptides is also responsible for the increased risk of psoriasis, as ACPA is not implicated in the pathogenesis of this inflammatory cutaneous disease. Even though the exact mechanism behind the increased risk of psoriasis among patients with periodontitis is unknown several possible explanations have been proposed. The first is associated with the common pathology between psoriasis and periodontitis, as excessive immune responses to the residing microbiota at the epithelial surface are identified in both conditions, which might suggest a common genetic predisposition that affects dendritic cells and TLR expression [43,94,95].

The other possible explanation involves the activation of Th17 cells and the increased expression of IL-17, that is a crucial cytokine in the pathogenesis of both psoriasis and psoriatic arthritis, induced by the bacteria involved in periodontal infection and their products $[43,95]$.

Reactive arthritis, previously known as Reiter's disease, is a pathological condition that shares characteristics with psoriasis and involves immune dysfunction, has also been associated with infectious triggers [96]. Consequently, it is possible that PD provides a similar infectious burden and acts as a trigger or risk factor for psoriasis.

Effects of confounding factors, such as gender, age, and smoking were controlled during the design of the current study. PD and psoriasis have not known risk factor $\mathrm{s}$ in common such the mentioned. Unknown and non measurable factors could lead to such an associations. However, the possible association examined between PD and psoriasis it is not clear if is causal or can be attributed to confounding effects. It is obvious that, prospective studies mainly are required in order to determine whether an association exists between both diseases. The present study was a first attempt to investigate the possible association between PD and psoriasis risk in a sample of Greek adults.

\section{Conclusion}

In conclusion, individuals with a higher BMI, excessive alcohol consumption, moderate/severe attachment loss and a number of missing teeth more than four were at significantly higher risk for developing psoriasis.

\section{References}

1. GudjonssonJE, Elder JT, Psoriasis. In: Wolff K, Goldsmith LA, Katz SI, Gilchrest BA, Paller AS, Leffell DJ. Fitzpatrick's dermatology in general medicine. 2008:169-94

2. Christophers E. Psoriasis-epidemiology and clinical spectrum. Clin ExpDermatol. 2001;26:314-320.

3. Vessey MP, Painter R, Powell J. Skin disorders in relation to oral contraception and other factors, including age, social class, smoking and body mass index. Findings in a large cohort study. Br J Dermatol. 2000; 143:815-820.

4. Setty AR, Curhan G, Choi HK. Obesity, waist circumference, weight change, and the risk ofpsoriasis in women: Nurses' Health Study II. Arch Intern Med. 2007;167:1670-1675.

5. Naldi L, Mercuri SR. Epidemiology of comorbidities in psoriasis. DermatolTher. 2010;23:114-118.

6. Mease PJ, Menter MA. Quality-of-life issues in psoriasis and psoriatic arthritis: outcome measures and therapies from a dermatological perspective. J Am AcadDermatol. 2006;54:685-704.

7. Qureshi AA, Dominguez PL, Choi HK, Han J, Curhan G. Alcohol intake and risk of incident psoriasis in US women: a prospective study. Arch Dermatol. 2010;146:1364-1369.

8. Armstrong AW, Harskamp CT, Dhillon JS, Armstrong EJ. Psoriasis and smoking:a systematic review and meta-analysis. Br J Dermatol. 2014;170:304-314.

9. Duffy DL, Spelman LS, Martin NG. Psoriasis in Australian twins. J Am Acad Dermatol.1993;29:428-434.

10. Christophers E. Comorbidities in psoriasis. Clin Dermatol. 2007; 25:529-534.

11. Preus HR, Khanifam P, Kolltveit K, Mørk C, Gjermo P. Periodontitis in psoriasis patients. Ablinded, case -controlled study.ActaOdontol 
Scand. 2010;68:165-170.

12. Telfer NR, Chalmers RJ, Whale K, Colman G. The role of streptococcal infection in the initiation of guttate psoriasis. Arch Dermatol. 1992;128:39-42.

13. Braun-Falco O, Plewig G, Wolff HH, Burgdorf WHC. Dermatology. Berlin: Springer-Verlag. 2000:585-607.

14. Albandar JM. Epidemiology and risk factors of periodontal diseases. Dent Clin North Am. 2005;49:517-532.

15. Albandar JM. Periodontal diseases in North America.Periodontol. 2002;29:31-69.

16. Pihlstrom BL, Michalowicz BS, Johnson NW. Periodontal disease. Lancet. 2005;366:1809-1820.

17. Albandar JM, Brunelle JA, Kingman A. Destructive periodontal disease in adults 30 years of age and older in the United States,1988-1994. J Periodontol. 1999;70:13-29.

18. Oliver RC, Brown LJ, Loe H. Periodontal diseases in the United States population. J Periodontol.1998;69:269-278.

19. Heitz-Mayfield LJA. Disease progression: Identification of high risk groups and individuals for periodontitis. J Clin Periodontol. 2005;32:196-209.

20. Kornman KS. Host modulation as a therapeutic strategy in the treatment of periodontaldisease. Clin Infect Dis. 1999;28:520-526.

21.Schaefer AS, Richter GM, Nothnagel M, Manke T, Dommisch H, Jacobs $\mathrm{G}$ et al. A genome-wide association study identifies GL T6D1 as a susceptibility locus for periodontitis. Hum Mol Genet. 2010;19:553562.

22. Bouchard P, Boutouyrie P, Mattout C, Bourgeois D. Risk assessment for severe clinical attachment loss in an adult population. J Periodontol. 2006;77:479-489.

23. Albandar JM. Global risk factors and risk indicators for periodontal diseases. Periodontol. 2000. 2002;29:177-206.

24. Genco RJ. Current view of risk factors for periodontal diseases.J Periodontol. 1996;67:1041-1049.

25. Borrell LN, Crawford ND. Socioeconomic position indicators and periodontitis: examining the evidence. Periodontology 2000. 2012;58:69-83.

26. Genco RJ, Borgnakke WS. Risk factors for periodontal disease. Periodontology 2000. 2013;62:59-94.

27. Susin C, DallaVecchia, Oppermann RV, Haugejorden O, AlbandarJM. Periodontal attachment loss in an urban population of Brazilian adults: effect of demographic, behavioral, and environmental risk indicators. J Periodontol. 2004;75:1033-1041.

28. Akhter R, Hannan MA, Okhubo R, Morita M. Relationship between stress factor and periodontal disease in a rural area population in Japan. Eur J Med Res. 2005;10:352-357.

29. Loos BG. Systemic markers of inflammation in periodontitis. J Periodontol.2005; 76: 2106-2115.

30. Ford PJ, Gamonal J, Seymour GJ. Immunological differences and similarities between chronic periodontitis and aggressive periodontitis.Periodontol. 2000; 53:111-123.

31. Brown LJ, Löe H. Prevalence, extent, severity and progression of periodontal disease. Periodontol 2000. 1993;2:57-71.

32. Kostic AD, Xavier RJ, Gevers D. The microbiome in inflammatory bowel disease: Current status and the future ahead. Gastroenterology. 2014;146:1489-1499.

33. Vogtmann E, Goedert JJ. Epidemiologic studies of the human micro biome and cancer. Br J Cancer. 2016;114:237-242.

34. Holmstrup P, Poulsen AH, Andersen L, Skuldbøl T, Fiehn NE. Oral infections and systemic diseases. Dent Clin North Am. 2003;47:575598.

35. Teng YT, Taylor GW, Scannapieco F, Kinane DF, Curtis M, Beck JD, et al. Periodontal health and systemic disorders. J Can Dent Assoc. 2002;68:188-192

36. Linden GJ, Lyons A, Scannapieco FA. Periodontal systemic associations: review of the evidence. J Periodontol. 2013;84:S8-S19.

37. Mercado FB, Marshall RI, Klestov AC, Bartold PM. Relationship between rheumatoid arthritis and periodontitis. J Periodontol. 2001;72:779-787.

38. de Pablo P, Dietrich T, McAlindon TE. Association of periodontal disease and tooth loss with rheumatoid arthritis in the US population. J Rheumatol. 2008;35:70-76.

39. Mikuls TR, Payne JB, Reinhardt RA, Thiele GM, Maziarz E, Cannella AC, et al. Antibody responses to Porphyromonasgingivalis (P.gingivalis) in subjects with rheumatoid arthritis and periodontitis. IntImmunopharmacol. 2009;9:38-42.

40. Leech MT, Bartold PM. The association between rheumatoid arthritis and periodontitis. Best Pract Res Clin Rheumatol. 2015; 29:189-201.

41. Pischon N, Pischon T, Kroger J, Gülmez E, Kleber BM, Bernimoulin JP, et al. Association among rheumatoid arthritis, oral hygiene and periodontitis. J Periodontol. 2008;79:979-986.

42. Cutler CW, Jotwani R. Dendritic cells at the oral mucosal interface. J Dent Res.2006;85:678-689.

43. Sabat R, Philipp S, Hoflich C, Kreutzer S, Wallace E, Asadullah K, et al. Immunopathogenesis of psoriasis. ExpDermatol. 2007;16:779-798.

44. Yamada J, Amar S, Petrungaro P. Psoriasis-associated periodontitis: a case report. J Periodontol. 1992;63:854-857.

45. Akazawa H, Nishimura F, Maeda H, Takashiba S, Mine A, Maekawa $\mathrm{K}$, et al. Regression of pustulosispalmaris et plantaris by periodontal treatment in a subject with severe periodontitis. Int J Dermatol. 2006; 45:1420-1422.

46. Sakiyama H, Kobayashi S, Dianzani U, Ogiuchi H, Kawashima M, Uchiyama T, et al. Possible involvement of T cell co-stimulation in pustulosispalmaris et plantaris via the induction of inducible costimulator in chronic focal infections. J Dermatol Sci. 2008;50:197207.

47. Hyman JJ, Reid BC. Epidemiologic risk factors for periodontal attachment loss among adults in the United States.J Clin Periodontol. 2003;30:230-237.

48. World Health Organization: Oral health surveys: basic methods. (4th Edn). Geneva: WHO.1997:47.

49. Lwanga SK, Lemeshow S. Sample size determination in health studies. A practical manual. Geneva: WHO. 1991.

50. Machuca G, Segura-Egea JJ, Jimenez-Beato G, Lacalle JR, Bullón P. Clinical indicators of periodontal disease in patients with coronary heart disease: A 10 years longitudinal study. Med Oral Patol Oral Cir Bucal. 2012;17:e569-574.

51. Molloy J, Wolff LF, Lopez-Guzman A, Hodges JS. The association of 
periodontal disease parameters with systemic medical conditions and tobacco use. J Clin Periodontol. 2004;31:625-632.

52. Cutress TW, Ainamo J, Sardo-Infrri J. The community periodontal index of treatment needs (CPITN) procedure for population groups and individuals. Int Dent J. 1987;37(4):222-233.

53. Wiebe CB, Putnins EE. The periodontal disease classification system of the American acaemy of periodontology an update. J Can Dent Assoc. 2000;66:594-597

54. Yoon HS, Wen W, Long J, Zheng W, Blot WJ, Cai Q. Association of Oral Health with Lung Cancer Risk in a Low Income Population of African Americans and European Americans in the Southeastern United States. Lung Cancer. 2019;127:90-95.

55. Vonneilich N, Jöckel KH, Erbel R, Klein J, Dragano N, Weyers S, et al. Does socioeconomic status affect the association of social relationships and health? A moderator analysis.Int J Equity Health. 2011;10:43.

56. Adisen E, Erduran F, Uzun S, Ali Guter M. Prevalence of smoking, alcohol consumption and metabolic syndrome in patients with psoriasis.Ann Bras Dermatol. 2018:93(2):205-211.

57. Department of Health. Sensible drinking: The report of an interdepartmental working group. 1995.

58. Poikolainen K, Reunala T, Karvonen J, Lauharanta J, Kärkkäinen P. Alcohol intake: a risk factor for psoriasis in young and middle aged men? Brit Med J. 1990;300(6727):780-783.

59. Gupta AK, Pandey SS, Pandey BL. Effectiveness of conventional drug therapy of plaque psoriasis in the context of consensus guidelines: a prospective observational study in 150 patients. Arch Dermatol. 2013;25(2):156-162.

60. Farkas A, Kemény L. Psoriasis and alcohol; is cutaneous ethanol one of the missing links. Br J Dermatol. 2010;162(4):711-716. doi:10.1111/ j.1365-2133.2009.09595.x

61. Yokoyama S, Hiramoto K, Koyama M, Ooi K. Chronic liver injury in mice promotes impairment of skin barrier function via tumor necrosis factor-alpha. CutanOculToxicol. 2016;35:194-203.

62. Parlet CP, Waldschmidt TJ, Schlueter AJ. Chronic ethanol feeding induces subset loss and hyporesponsiveness in skin T cells. Alcohol Clin Exp Res. 2014;38(5):1356-1364.

63. Thompson MG, Navarro F, Chitsike L, Ramirez L, Kovacs EJ, Watkins SK. Alcohol exposure differentially effects anti-tumor immunity in females by altering dendritic cell function. Alcohol. 2016;57:1-8.

64. Parlet CP, Schlueter AJ. Mechanisms by which chronic ethanol feeding impairs the migratory capacity of cutaneous dendritic cells. Alcohol Clin Exp Res. 2013;37(12):2098-2107.

65. Harvima I, Nilsson G, Suttle M, Naukkarinen A. Is there a role for mast cells in psoriasis? Arch Dermatol Res. 2008;300(9):461-478.

66. Zimatkin SM, Anichtchik OV. Alcohol-histamine interactions.Alcohol. 1999;34(2):141-147.

67. Mendes LO, Amorim JP, Teixeira GR, Chuffa LGA, Fioruci BA, Pimentel TA, et al. Mast cells and ethanol consumption: interactions in the prostate, epididymis and testis of UChBrats. Am J ReprodImmunol. 2011;66(3):170-178.

68. Caroline Svanström, Sol-Britt Lonne-Rahm, KlasNordlind. Psoriasis and alcohol.Psoriasis Auckl). 2019;9:75-79.

69. Hercogová J, Ricceri F, Tripo L, Lotti T, Prignano F. Psoriasis and body mass index. DermatolTher 2010;23(2):152-154.
70. Nakamizo S, Honda T, Kabashima K. Obesity and inflammatory skin diseases. Trends Immunother. 2017;1:67-74.

71. Sbidian E, Chaimani A, Garcia-Doval I, Do G, Hua C, Mazaud C, Droitcourt C, et al. Systemic pharmacological treatments for chronic plaque psoriasis: a network meta-analysis. Cochrane Database Syst Rev. 2017;12: CD011535.

72. Dowlatshahi EA, van derVoort EA, Arends LR, NijstenT. Markers of systemic inflammation in psoriasis: a systematic review and metaanalysis. Br J Dermatol. 2013;169: 266-282.

73. Stjernholm T, Ommen P, Langkilde A, Johansen C, IversenL, Rosada C, et al. Leptin deficiency in mice counteracts imiquimod (IMQ)-induced psoriasis-like skin inflammation while leptin stimulation induces inflammation in human keratinocytes. ExpDermatol. 2017;26:338345.

74. Davidovici BB, Sattar N, Jörg PC, Puig L, Emery P, Barker JN, et al. Psoriasis and systemic inflammatory diseases: Potential mechanistic links between skin disease and co-morbid conditions. J Invest Dermatol. 2010;130:1785-1796.

75. Fadel HT, Flytström I, Calander AM, Bergbrant IM, Heijl L, Birkhed D. Profiles of Dental Caries and Periodontal Disease in Individuals with or without Psoriasis. J Periodontol. 2013;84(4):477485.

76. Ungprasert P, Wijarnpreecha K, Wetter DA. Periodontitis and Risk of Psoriasis: A Systematic Review and Meta-analysis. J EurAcadDermatolVenereol. 2017;31(5):857-862.

77. Keller JJ, Lin HC. The effects of chronic periodontitis and its treatment on the subsequent risk of psoriasis. Br J Dermatol. 2012;167:13381344.

78. Lazaridou E, Tsikrikoni A, Fotiadou C, Kyrmanidou E, Vakirlis E, Giannopoulou C, et al. Association of chronic plaque psoriasis and severe periodontitis: a hospital based case-control study. J EurAcadDermatolVenereol. 2013;27:967-972.

79. Skudutyte-Rysstad R, Slevolden EM, Hansen BF, Sandvik L, Preus HR. Association between moderate to severe psoriasis and periodontitis in a Scandinavian population. BMC Oral Health. 2014;14:139.

80. Ganzetti G, Campanati A, Santerlli A, Pozzi V, Molinelli E, Minnetti I, et al. Involvement of the oral cavity in psoriasis: results of a clinical study. Br J Dermatol. 2015;172:282-284.

81. Nakib S, Han J, Li T, Joshipura K, Qureshi AA. Periodontal disease and risk of psoriasis among nurses in the United States. ActaOdontol Scand. 2013;71(6):1423-1429.

82. Gamonol J, Acevedo A, Bosnones A. Levels of IL-1 beta , 8, and 10 and rantes in gingival crevicular fluid and cell populations in adult periodontitis patients and the effect of periodontal treatment. J Periodontol 2000;71:1535-1545.

83. Ishihara Y, Nishihara T. Kuroyaragi T. Gingival crevicular fluid IL1 and IL-1 receptor antagonist levels in periodontally healthy and diseased sites. J Periodontal Res. 1997;32:524-529.

84. Van deKerkhof PC. The Woronoff zone surrounding the psoriatic plaque. Br J Dermatol. 1998;139:167.

85. Grossi SG, Genco RJ. Periodontal disease and diabetes mellitus: a twoway relationship. Ann Periodontol 1998;3:51-61.

86. Forner L, Larsen T, Kilian M, Holmstrup P. Incidence of bacteremia after chewing, tooth brushing and scaling in individuals with 
periodontal inflammation. J Clin Periodontol. 2006;33:401-407.

87. Ortega C, Fernández-A S, Carrillo JM, Romero P, Molina IJ, Moreno JC, et al. IL-17 producing CD8+ T lymphocytes from psoriasis skin plaques are cytotoxic effector cells that secrete Th17-related cytokines. J Leukoc Biol. 2009; 86:435-443.

88. Behfarnia P, Birang R, Andalib AR, Asadi S. Comparative Evaluation of IFN $\gamma$, IL4 and IL17Cytokines in Healthy Gingiva and Moderate to Advanced Chronic Periodontitis. Dent Res J.(Isfahan). 2010;7:45-50.

89. Candia L, Marquez J, Hernandez C, Zea AH, Espinoza LR. Toll-like receptor-2 expression is upregulated in antigenpresenting cells from patients with psoriatic arthritis: a pathogenic role for innate immunity? J Rheumatol. 2007; 34:374-379.

90. Mahanonda R, Pichyangkul S. Toll-like receptors and their role in periodontal health and disease. Periodontol 2000. 2007;43:41-55.

91. Burns E, Bachrach G, Shapira L, Nussbaum G. Cutting Edge:TLR2 is required for the innate response to Porphyromonasgingivalis: activation leads to bacterial persistence and TLR2deficiency attenuates induced alveolar bone resorption. J Immunol. 2006;177:8296-8300.

92. Hurst J, von Landenberg P. Toll-like receptors and autoimmunity. Autoimmune Rev. 2008; 7:204-208.

93. Mikuls TR, Payne JB, Deane KD, Thiele GM. Autoimmunity of the lung and oral mucosa in a multisystem inflammatory disease: The spark that lights the fire in rheumatoid arthritis? J Allergy Clin Immunol. 2016;137:28-34.

94. Sharma A, Raman A, Pradeep AR. Association of chronic periodontitis and psoriasis: periodontal status with severity of psoriasis. Oral Dis. 2015;21:314-319.

95. Ebersole JL. Humoral immune responses in gingival crevice fluid: local and systemic implications. Periodontol 2000. 2003;31:135-166.

96. Wu IB, Schwartz RA. Reiter's syndrome: the classic triad and more. J Am AcadDermatol. 2008; 59:113-121. 